\title{
Introduction to Quantum Groups
}


This page is intentionally left blank 


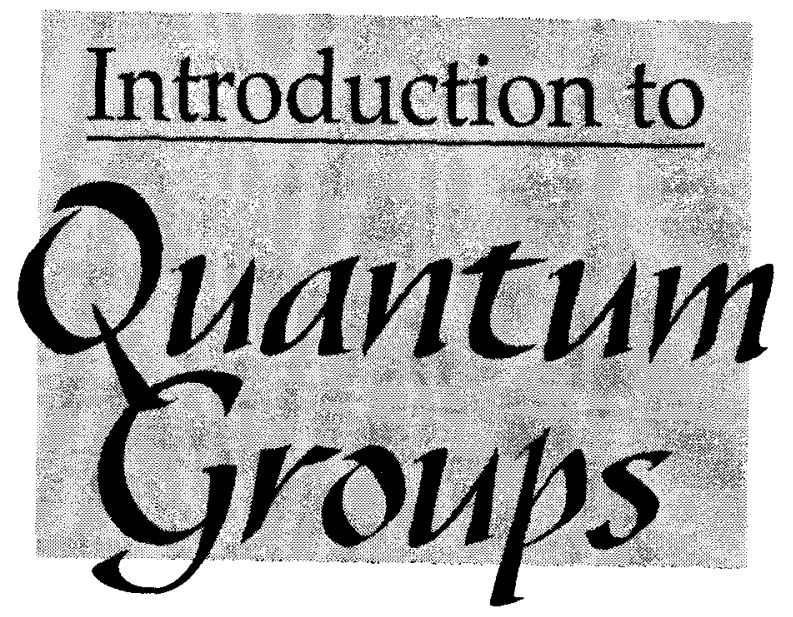

\section{Chaichian}

High Energy Physics Division

University of Helsinki and

Physics Research Institute

Helsinki

\section{A. Demichev}

Nuclear Physics Institute

Moscow State University

and

Centro Brasileiro de Pesquisas Fisicas

Rio de Janeiro 


\title{
Published by
}

World Scientific Publishing Co. Pte. Ltd.

P O Box 128, Farrer Road, Singapore 912805

USA office: Suite 1B, 1060 Main Street, River Edge, NJ 07661

UK office: 57 Shelton Street, Covent Garden, London WC2H 9HE

\author{
Library of Congress Cataloging-in-Publication Data \\ Chaichian, M. (Masud), 1941- \\ Introduction to quantum groups / by M. Chaichian and A. P. Demichev. \\ 356 p. $22 \mathrm{~cm}$. \\ Includes bibliographical references and index. \\ ISBN 9810226233 \\ 1. Quantum groups. I. Demichev, A. P. (Andrei Pavlovich) \\ II. Title. \\ QC20.7.G76C47 1996 \\ 530.1'5255--dc20 \\ 96-25942 \\ CIP
}

\section{British Library Cataloguing-in-Publication Data}

A catalogue record for this book is available from the British Library.

Copyright $\odot 1996$ by World Scientific Publishing Co. Pte. Ltd.

All rights reserved. This book, or parts thereof, may not be reproduced in any form or by any means, electronic or mechanical, including photocopying, recording or any information storage and retrieval system now known or to be invented, without written permission from the Publisher.

For photocopying of material in this volume, please pay a copying fee through the Copyright Clearance Center, Inc., 222 Rosewood Drive, Danvers, MA 01923, USA. In this case permission to photocopy is not required from the publisher.

This book is printed on acid-free paper.

Printed in Singapore by Uto-Print 


\section{To our parents}


This page is intentionally left blank 


\section{Preface}

The importance of the theory of Lie groups and algebras for theoretical physics can hardly be disputed. Its applications in most branches of modern physics have proved to be extremely fruitful not only for the solution of already existing problems but also as a guide for the formulation and development of essentially new ideas and approaches for the description of physical phenomena. It turns out, however, that the present level of theoretical problems requires more general algebraic objects called quantum groups which contain Lie groups and Lie algebras as particular and, in a sense, degenerate cases. The mathematical theory of these new objects arose as an abstraction from constructions developed in the frame of the inverse scattering method of solution of quantum integrable models. This origin implied, from the very beginning, the importance of this mathematical theory for physics. On the other hand, the theory has proved to be so rich and powerful that it seems natural to apply it to different problems far beyond the original area, generalizing such basic physical notions as space-time symmetries, gauge symmetries, different types of dynamical symmetries and so on.

At present the theory being highly developed, is still far from full completeness. Its mathematical foundation are summarized in a number of books $[171,64,61,137,174]$. But many results, especially concerning physical appli- 
cations, are scattered in a variety of original papers and reviews, often rather difficult for a first reading.

In this book we have aimed at giving an introduction to some of the principal ideas of the quantum group theory accompanied by examples of its different applications, assuming that the reader is familiar with the theory of Lie groups, Lie algebras and quantum mechanics. A certain acquaintance with some basic facts from the theory of supergroups and quantum field theory is also assumed. Actually, the latter is important only for a better understanding of some applications of the new mathematics. Though the most natural applications of quantum groups are in the theory of integrable systems, we have not concentrated on this subject. The main reason is that we have intended to show a variety and diversity of possible applications of quantum groups, including the theory of elementary particles and field theory. Besides, the inverse scattering method is a very extensive subject which cannot be reduced only to quantum groups. Its self-contained exposition would require an essential increase of the volume of the book and would lead us away from the main topic.

By now quantum groups and, in a more general sense, non-commutative geometry have become an immense and versatile subject. We have selected certain topics for discussion and we are well aware of the fact that many important topics have been either left out or are only mentioned briefly. We hope that partially this is compensated by references in our book to the original papers and appropriate reviews. Also we have tried to use a minimum of mathematical tools. Thus proofs of a number of theorems and details of applications are either briefly sketched or omitted, adequate references being given to enable the interested reader to study the subject in full completeness.

In writing this book we have benefited from discussions on various topics with many of our colleagues, in particular with Peter Prešnajder. We thank all of them for useful discussions and for their advice. Especially, it is a pleasure to express our gratitude to Peter Kulish and to acknowledge stimulating discussions, suggestions and criticism. We are deeply indebted for all what he has offered to us. We are also grateful to Wen-Feng Chen for making many improvements in the manuscript. A.D. is much grateful to José Helayel-Neto for constant support and to the Brazilian Research Council and INTAS for financial support. 


\section{Contents}

$\begin{array}{ll}\text { Preface } & \text { vii }\end{array}$

Introduction 1

Notational Conventions. . . . . . . . . . . . 5

1 Mathematical Aspects of Quantum Group Theory and NonCommutative Geometry

1.1 Non-commutative algebras, differential calculi, transformations and all that $\ldots \ldots \ldots \ldots \ldots \ldots \ldots \ldots$

1.2 Hopf algebra and Poisson structure of classical Lie groups and

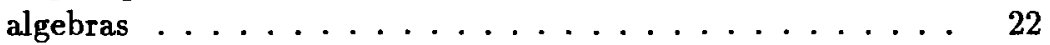

1.3 Deformation of co-Poisson structures . . . . . . . . . 41

1.4 Quasi-triangular Hopf algebras and quantum double construction 52

1.5 Quantum matrix groups . . . . . . . . . . . 60

1.5.1 Quantum groups $G L_{q}(n)$ and $S L_{q}(n) \ldots \ldots \ldots \ldots 62$

1.5.2 Quantum groups $S O_{q}(N)$ and $S p_{q}(n) \ldots \ldots \ldots \ldots 66$

1.5.3 Twists of quantum groups and multiparametric defor-

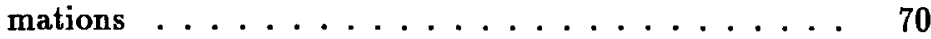

1.6 Quantum deformation of differential and integral calculi . . . 77

1.6.1 Differential calculus on q-groups . . . . . . . . . 78

1.6.2 Differential calculus on quantum spaces . . . . . . . 81

1.6.3 q-Deformation of integral calculus . . . . . . . 85

1.7 Elements of quantum group representations $\ldots \ldots \ldots \ldots 92$

1.7.1 Corepresentations of quantum groups . . . . . . . 9 94

1.7.2 Representations of quantum universal enveloping algebras 99

1.7.3 Representations of quantized algebras of functions . . 107 
2 q-Deformation of Harmonic Oscillators, Quantum Symmetry and All That

2.1 q-Deformation of single harmonic oscillator . . . . . . . . . 112

2.2 Bargmann-Fock representation for q-oscillator algebra in terms of operators on quantum planes . . . . . . . . . . 126

2.3 Quasi-classical limit of q-oscillators and q-deformed path integrals

2.3.1 Quasi-classical limit of q-oscillators (with real parameter of deformation) . . . . . . . . . . . 133

2.3.2 Path integral for q-oscillators (real q) . . . . . . . 143

2.3.3 Path integral for q-oscillators with root of unity value of deformation parameter . . . . . . . . . . 146

2.4 q-Oscillators and representations of QUEA . . . . . . . . . 151

2.4.1 q-Deformed Jordan-Schwinger realization . . . . . . . 151

2.4.2 Quantum Clebsch-Gordan coefficients and Wigner-Eckart theorem . . . . . . . . . . . . 153

2.4.3 Covariant systems of q-oscillators . . . . . . . . 158

2.5 q-Deformation of supergroups and conception of braided groups 166

2.5.1 q-Supergroups, q-superalgebras and q-superoscillators . 166

2.5.2 Braided groups and spaces . . . . . . . . . . . 171

2.6 Quantum symmetries and $q$-deformed algebras in physical systems ...................... 177

2.6.1 Integrable one-dimensional spin-chain model . . . . . 177

2.6.2 A model in quantum optics . . . . . . . . . . 182

2.6.3 Magnetic translations and the algebra $s l_{q}(2) \ldots \ldots 183$

2.6.4 Pseudoeuclidian quantum algebra as symmetry of phonons .................. 185

2.6.5 q-Oscillators and regularization of quantum field theory 188

2.6.6 q-Deformed statistics and the ideal q-gas . . . . . . 192

2.6.7 Nonlinear Regge trajectory and quantum dual string theory . . . . . . . . . . . . . . 198

2.6.8 q-Deformation of the Virasoro algebra . . . . . 203

3 q-Deformation of Space-Time Symmetries 209

3.1 One-dimensional lattice and $q$ - deformation of differential calculus ......................... 210

3.2 Multidimensional Jackson calculus and particle on two-dimensional quantum space . . . . . . . . . . . . . 214

3.3 Projective construction of quantum inhomogeneous groups .. 224 
3.4 Twisted Poincaré group and geometry of $q$-deformed Minkowski space . . . . . . . . . . . . . . . 229

3.4.1 Quantum deformation of the Poincaré group . . . . . 230

3.4.2 Quantum Minkowski geometry . . . . . . . . . 234

3.4.3 q-tetrades and transformation to commuting coordinates 237

3.4.4 Twisted Poincaré algebra and induced representations of the q-group . . . . . . . . . . . . . . 239

3.4.5 Twisted Minkowski space in the case of related $q$ and $\hbar$ constants ................... 244

3.5 Jordan-Schwinger construction for q-algebras of space-time symmetries and contraction of quantum groups . . . . . . . 246

3.5.1 Fock space representation of the q-Lorentz algebra . . . 247

3.5.2 q-Deformed anti-de Sitter algebra and its contraction - 249

3.5.3 Quantum inhomogeneous groups from contraction of $\mathbf{q}-$ deformed simple groups . . . . . . . . . 256

3.6 Elements of general theory of $q$-inhomogeneous groups and classification of q-Poincaré groups and q-Minkowski spaces .... 3.6.1 Classification of $\mathrm{q}$-Lorentz groups and $\mathrm{q}$-Minkowski

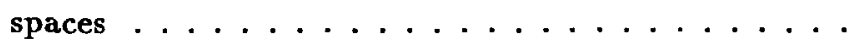

3.6.2 General definition and properties of inhomogeneous quantum groups . . . . . . . . . . . . 268

3.6.3 Classification of quantum Poincaré groups . . . . . 271

4 Non-commutative Geometry and Internal Symmetries of Field Theoretical Models

4.1 Non-commutative geometry of Yang-Mills-Higgs models . . . . 283

4.2 Posets, discrete differential calculus and Connes-Lott-like models . . . . . . . . . . . . . . . . . . 294

4.2.1 Yang-Mills-Higgs theory from dimensional reduction . . 294

4.2.2 Finite approximation of topological spaces . . . . . 296

4.3 Basic elements of quantum fibre bundle theory . . . . . . . 307

Appendix: Short Glossary of Selected Notions from the Theory of Classical Groups 